\title{
Development of an analytical diffusion model for modeling hydrogen isotope exchange
}

\author{
J.L. Barton*, Y.Q. Wang a , R.P. Doerner, G.R. Tynan \\ University of California San Diego, 9500 Gilman Drive, La Jolla, CA, 92093-0417, USA \\ ${ }^{a}$ Los Alamos National Laboratory, P.O. Box 1663, Los Alamos, NM, 87545, USA
}

\begin{abstract}
We create a model for $\mathrm{H}$ retention depth profiles in $\mathrm{W}$ and subsequently model how this profile changes after isotope exchange. This is accomplished by calculating how trapping defects in $\mathrm{W}$ accumulate $\mathrm{D}$ (or $\mathrm{H}$ ) inventory as $\mathrm{W}$ is being exposed to plasma. Each trapping site is characterized by a trapping rate and a release rate, where the only free parameters are the distribution of these trapping sites in the material. The filled trap concentrations for each trap type are modeled as a diffusion process because post-mortem deuterium depth profiles indicate that traps are filled well beyond the ion implantation zone $(2-5 \mathrm{~nm})$. Using this retention model, an isotope exchange rate is formulated. The retention model and isotope exchange rate are compared to low temperature isotope exchange experiments in tungsten with good agreement. The limitations of the current model highlight important physics and motivate future work.
\end{abstract}

PACS: 52.40.Hf, 61.72.Qq

PSI-21 keywords: Deuterium inventory, Tritium inventory, Tungsten, Nuclear reaction analysis

*Corresponding author address: 9500 Gilman Drive \#0417, La Jolla, CA 92093, USA.

*Corresponding author e-mail: jbarton@ucsd.edu

Presenting Author: Joseph Barton

Presenting Authore-mail: jbarton@ucsd.edu 


\section{Introduction}

Hydrogenic species retention in plasma facing materials (PFCs) is a major concern for practical operation of magnetically confined fusion reactors [1]. Tritium fuel accumulates in PFCs diffusing well beyond their implantation depth of 2-5 nm, raising both safety and tritium self-sufficiency concerns. The isotope exchange technique will be useful in prolonging the lifetime of PFCs because much of the trapped tritium fuel in PFCs can be recycled back into the plasma. By treating a PFC that has $\mathrm{H}$ isotopes retained within it using a plasma of another $\mathrm{H}$ isotope, it has been observed [2-4] that the retained $\mathrm{H}$ isotopes are exchanged out of the material, even beyond the ion implantation depth of a few nanometers. An isotope exchange model was proposed [5] that depended on the ion implantation energy for materials like $\mathrm{Si}, \mathrm{C}$, and other Ti composites. However, there is no predictive model for the isotope exchange rate in materials like $\mathrm{W}$, where the arrival rate of the exchanging $\mathrm{H}$ isotope at depths beyond the ion implantation zone is due to $\mathrm{H}$ isotope diffusion and not energetic deposition. This project seeks to create a model for $\mathrm{H}$ retention depth profiles in $\mathrm{W}$ and subsequently model how this profile changes due to isotope exchange.

\section{Experimental}

The metal samples used in these experiments were $2 \mathrm{~mm}$ thick by $6 \mathrm{~mm}$ diameter hotrolled tungsten with purity of 99.97 wt.\% manufactured by Plansee [6]. These samples were polished mechanically to a mirror-like finish, cleaned in an acetone and ethanol ultrasonic bath, and annealed at $1200 \mathrm{~K}$ for one hour to remove any impurities and reduce other intrinsic defects.

A radio frequency (rf) helicon wave plasma source, characterized in [7], was used to treat the $\mathrm{W}$ samples with $\mathrm{D}$ and $\mathrm{H}$ plasmas. With an input power of $1.2 \mathrm{~kW}$, plasma densities range from $6-9 \times 10^{16} \mathrm{~m}^{-3}$, and plasma fluxes are $1.5( \pm .2) \times 10^{20} \mathrm{ions} / \mathrm{m}^{2} / \mathrm{s}$, both measured by 
sweeping a Langmuir probe in front of the sample. The flux was also verified by measuring the current to the sample holder. The ion energy in these experiments was $100 \mathrm{eV}$, which was estimated from subtracting the negative bias on the sample holder from the measured plasma potential. Each W sample was treated with D plasma to a fluence of $10^{24}$ ions $/ \mathrm{m}^{2}$. Then the vacuum chamber was pumped down for 5 minutes, the gas inlet lines were purged with $\mathrm{H}_{2}$ gas, and the sample was treated with $\mathrm{H}$ plasma to a fluence of either $10^{22}, 5 \times 10^{22}$, $10^{23}$, or $10^{24}$ ions $/ \mathrm{m}^{2}$, except the control sample which was not exposed to $\mathrm{H}$ plasma. The temperature of the samples during plasma treatment was measured using a thermocouple pressed to back of the sample, insulated from the plasma. Samples were held at a constant $370 \mathrm{~K}$ throughout plasma exposure.

The retained D concentration profile was measured using nuclear reaction analysis (NRA) by the $\mathrm{D}\left({ }^{3} \mathrm{He}, \mathrm{p}\right) \alpha$ reaction at the Ion Beams Materials Laboratory (IBML) at Los Alamos National Laboratory (LANL). The measurement technique is described in more detail elsewhere [8]. Tungsten samples were irradiated with a ${ }^{3} \mathrm{He}$ ion beam at room temperature with energies of $0.5,0.8,2,2.75,3.5$, and $4.5 \mathrm{MeV}$ to produce a $\mathrm{D}$ depth profile with a depth resolution of less than one micron up to a depth of $7.7 \mu \mathrm{m}$, similar to [2].

\section{Model}

\subsection{Retention}

$\mathrm{H}$ isotopes are retained in defects in the crystalline lattice as they diffuse in solution through $\mathrm{W}$ into the bulk region, which is defined as greater than the implantation zone of the plasma ions. The diffusion of $\mathrm{H}$ in solution has been studied extensively through modelling and experiment [9], but we want to study how particular types of defects, such as dislocations or vacancies, accumulate inventory as $\mathrm{H}$ diffuses through $\mathrm{W}$. We will assume that these defects do not migrate so that the arrival rate of atoms to a trap site (i.e. the filling evolution of the trap site) will be related to the measured diffusion coefficient of solute $\mathrm{H}$ in $\mathrm{W}$. 
Formulating the problem as the evolution of filled trap site concentrations, as opposed to the diffusion of solute atoms explicitly, allows for easy comparison to the trapped D spatial profile in NRA experiments. $\mathrm{H}$ atom interactions with defects will be defined by a trapping and release rate, similar to the diffusion code TMAP [10]. The trapping rate for each diffusing atom to the $i^{\text {th }}$ trap site is

$\alpha_{t, i}=\frac{D_{s}}{\lambda^{2}}\left(\frac{C_{t, i}^{0}-C_{t, i}}{N_{W}}\right)$,

where the subscript $i$ refers to the $i^{\text {th }}$ trapping energy discussed below; $D_{S}$ is the diffusion coefficient of atoms through solution defined by [11]; $\lambda$ is the lattice parameter; $C_{t, i}^{0}$ is the total concentration of traps of type $i$, which we will assume to be uniformly distributed in the material without space or time dependence; $C_{t, i}$ is the concentration of filled traps of type $i$ and does depend on space and time; and $N_{W}$ is the atomic density of W. The choice of the value of $D_{s}$, the Frauenfelder coefficient, is worthy of mention because we seek to decouple the effects of trapping from solute diffusion. Frauenfelder's measurement of $D_{s}$ at temperatures greater than $1000 \mathrm{~K}$ is assumed to have little trapping effects. We extrapolated this value of $D_{s}$ to lower temperatures to reflect only the solute diffusion and treat trapping separately. The coefficient of (1) defines the lattice jump rate of $\mathrm{H}$ in $\mathrm{W}$, and this is multiplied by the probability of finding an empty trap of type $i$. This probability is not constant because $C_{t, i}$ increases until it equals $C_{t, i}^{0}$, at which point the trapping rate is reduced to zero. The release rate of the $i^{\text {th }}$ trap site is

$\alpha_{r, i}=v_{D} e^{-E_{t, i} / T}$,

where $v_{D}$ is the maximum frequency of oscillations in one dimension due to thermal vibrations (Debye frequency) of the trapped atom, $E_{t, i}$ is the energy the trapped atom has to gain to escape the trap site, and $T$ is the temperature of $\mathrm{W}$ in energy units. These rate coefficients (1-2) can be used to model one atom in each trap. However, density functional 
theory (DFT) models have shown [12-14] that trapping defects in W have a finite capacity for $\mathrm{H}$ isotopes, but do not necessarily have a limit of one atom. For instance, it has been modeled that one vacancy defect in $\mathrm{W}$ can accumulate up to $10 \mathrm{H}$ atoms [12]. But as the defect accumulates more atoms, the energy $E_{t, i}$ the trapped atom must gain to escape the trap decreases (i.e. a single $\mathrm{H}$ atom in a vacancy must gain more energy to escape than an $\mathrm{H}$ atom with nine other $\mathrm{H}$ atoms in that vacancy). We handle these various trapping energies by treating them as separate trapping sites: A vacancy with no $\mathrm{H}$ atoms is treated as a separate trap from a vacancy with 1 trapped $\mathrm{H}$ atom, which is separate from a vacancy with 2 trapped $\mathrm{H}$ atoms and so on. The trapping energy must then be a function of the number of trapped atoms, estimated from the DFT models. In this way, we can include the fact that defects in $\mathrm{W}$ can hold more than one $\mathrm{H}$ atom. By treating each trapping energy as a separate trapping site, we can use the trapping and release rates to model one atom in each trap site.

Since $\mathrm{H}$ isotopes must first diffuse through solution to reach a trap site, we treat the concentration of each partially filled trap site as a diffusive process,

$\frac{\partial C_{t, i}}{\partial t}=D_{s} \frac{\partial^{2} C_{t, i}}{\partial x^{2}}-\frac{\partial C_{s}^{\text {local }}}{\partial t}$,

where the use of $D_{s}$ in this equation is due to the evolution of the filled trap sites depending on the arrival of solute atoms and $C_{s}^{\text {local }}$ is local concentration of this $\mathrm{H}$ isotope in solution. Equation (3) defines the concentration of a partially filled trap of type $i$ in terms of a one dimensional diffusion process, combined with a volumetric loss rate due to the escape of trapped atoms which enter into solution. The sum of all the solutions of (3) over all of the $i$ trap types will then give the total retention profile that can be compared to NRA data. We

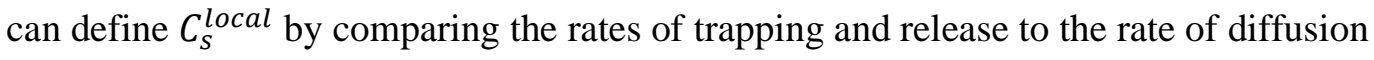
through solution over a relevant length scale. Figure 1 shows that the diffusion rate (over a length scale of $10 \mu \mathrm{m}$ ) is much less than the release rate (with a trapping energy of $1 \mathrm{eV}$, similar to a vacancy trap [12]) and the trapping rate over all temperature ranges for fusion 
related plasma-material experiments. As the concentration of filled traps increases toward saturation (i.e. $C_{t, i} / C_{t, i}^{0} \rightarrow 1$ ), the trapping rate decreases, approaching the diffusion rate.

However, the release rate is constant with constant material temperature, making the trapping rate's approach to saturation slow as the release rate becomes much greater than the trapping rate. Therefore, we will make the assumption that the trapping and release rates are always much greater than the diffusion rate throughout the simulation. This assumption means that we can write down a local equilibrium condition between the concentration of trapped atoms and the local concentration of atoms in solution,

$\alpha_{t, i} C_{s}^{\text {local }}=\alpha_{r, i} C_{t, i}$.

Solving this equation for $C_{s}^{\text {locl }}$ with the definitions (1-2) and plugging it into equation (3), we can then find the rate at which the concentration of atoms in trap type $i$ fills throughout the material.

Solutions for each trap type $i$ of equation (3) require initial and boundary conditions. Initially the concentration of trapped $\mathrm{D}$ (or $\mathrm{H}$ ) is assumed to be zero throughout the domain, i.e. we assume initially that $C_{t, i}=0$ for all $i$ and all position $x$. The boundary conditions are then

$C_{t, i}(x=0, t>0)=C_{t, i}^{0}$

$C_{t, i}(x \rightarrow \infty, t>0)=0$.

Condition (5) states that, on the surface, trap type $i$ becomes completely saturated, which is reasonable given the high fluxes in most plasma experiments. For convenience, we will assume that the NRA measurement is taken instantaneously after the plasma is turned off (i.e. the trapped concentrations are frozen in at the end of the experiment) so that the transport of atoms after plasma exposure are not calculated. H isotopes can diffuse to the surface and desorb after plasma treatment, but we are taking this effect to be small. Frauenfelder's diffusion coefficient [11] at our experimental temperatures estimates that the deepest 
penetration of hydrogen isotopes through solution is $90 \mu \mathrm{m}$, and our sample thickness is 2 $\mathrm{mm}$. So it is reasonable to have boundary condition (6) such that no traps are filled for very large depths. By writing the initial and boundary conditions in this way, we can combine the space and time variables into one variable, and hence solve an ordinary differential equation, using the Boltzmann transformation

$y=\frac{x}{2 \sqrt{D t}}$

where $D=D_{s} /\left(1+\frac{\alpha_{r, i} \lambda^{2} N_{W}}{D_{S} C_{t, i}^{0}}\right)$ is the effective diffusion coefficient. This effective diffusion coefficient reflects the decoupling of solute diffusion from the effects of trapping. Equation (3) can now be solved using a simple numerical method because of the non-linearity caused by the non-constant value of the trapping rate. Figure 2 shows the numerical solution for one trap type after exposure to D plasma (blue curve) for $t=5000 \mathrm{~s}$. To first order, the trapping rate is constant. In that case, the solutions of (3) are complementary error functions.

To model our NRA data, we chose three trap types with a total of 21 trapping energies. According to Liu's DFT work [12], a vacancy within a grain (or bulk vacancy) needs to be modeled with 10 trapping energies because up to 10 atoms can occupy this defect. Dislocation defects can hold up to 6 atoms and therefore have 6 trapping energies, according to a model proposed by Xiao [13]. Finally, Zhou [14] proposes 5 grain boundary (GB) vacancies that can hold only 1 atom each but have different trapping energies based on the lattice configuration. Because all of the trapping energies are defined, the release rate is therefore completely constrained. We then define the distribution of these 3 defect types $\left(C_{t, i}^{0}\right)$ as fitting parameters for the retention data. We chose uniform distributions of 0.04 , $1.3 \times 10^{-3}$, and $5 \times 10^{-4}$ at. $\% C_{t}^{0} / N_{W}$ for GB, dislocation, and bulk vacancy traps, respectively, which qualitatively represent the occurrence of these defects. We chose GB defects to be the most prominent inherent defect in our polycrystalline $\mathrm{W}$ material, then chose dislocations as 
the next largest defect due to another retention model fit [15], and inherent bulk vacancies were chosen as the least prominent defect. All parameters (trap concentrations and energies) are now fixed when used to calculate the isotope exchange rate.

\subsection{Isotope exchange}

An isotope exchange model was proposed by Doyle [5] where the ion implantation energy of $\mathrm{H}$ isotopes into $\mathrm{Si}, \mathrm{C}$, and other Ti composites was the key variable that governed the trapping physics. Since experiments with $\mathrm{W}$ have shown that isotope exchange happens at micrometers into the bulk, which is much greater than plasma ion implantation depth of a few nanometers [2-4], this model needs to be adapted to a case in which the diffusion of an atom resulting from ion implantation is included. Doyle's model says that each isotope has an equal probability of being trapped, and whichever atom is not trapped then disappears from the material. The loss rate, say, of $\mathrm{D}$ being exchanged by $\mathrm{H}$ is proportional to the probability of $\mathrm{H}$ taking the $\mathrm{D}$ filled trap times the arrival rate of $\mathrm{H}$. Therefore, we have for the loss rate of $\mathrm{D}$ being exchanged by $\mathrm{H}$

$\frac{\partial C_{t, i}^{D}}{\partial t}=-\frac{1}{2} \frac{C_{t, i}^{D}}{C_{t, i}^{0}} \frac{\partial C_{s}^{H, l o c a l}}{\partial t}$,

where the $\mathrm{D}$ and $\mathrm{H}$ superscripts indicate either trapped concentrations of $\mathrm{D}$ or $\mathrm{H}$, respectively. The one half in (8) is the probability that $\mathrm{H}$ will exchange with $\mathrm{D}$, since our model states that only one atom can occupy each trap site and has two competing atoms. The second ratio is the fraction of traps of type $i$ that are filled by D. The local equilibrium condition (4) relies on the fact that atoms are trapped and released orders magnitude faster than they can diffuse through solution. This is true for both isotopes. During the isotope exchange phase, we only consider the diffusion of the exchanging isotope $(\mathrm{H})$. We do not consider the diffusion of D atoms during the $\mathrm{H}$ plasma treatment, but we do consider trapped $\mathrm{D}$ atoms to be in equilibrium with solution as before. Equation (4) still applies to D atoms that have not been exchanged, but if an $\mathrm{H}$ atom has diffused to that trap site, an $\mathrm{H}$ atom has a fifty percent 
chance of occupying trap instead. We could alternatively model how D atoms diffuse and are re-trapped during isotope exchange by solving the reaction-diffusion equations for both species simultaneously. However, this first simple model attempts to extract some of the basic physics of isotope exchange. Due to the flood of $\mathrm{H}$ atoms occupying solution during the exchange phase, we assume that $\mathrm{D}$ atoms are not trapped again after exchanging. Equation (8) can then be solved directly to find the concentration of D after isotope exchange with $\mathrm{H}$

$C_{t, i}^{D}=C_{t, i}^{D, i n i t} \exp \left\{A\left[1-\left(1-\frac{C_{t, i}^{H}}{C_{t, i}^{0}}\right)^{-1}\right]\right\}$

where $C_{t, i}^{D, i n i t}=C_{t, i}\left(x, t=t_{D}\right)$ is the $\mathrm{D}$ filled trap profile after exposure to $\mathrm{D}$ plasma for time $t_{D}, A=\frac{\alpha_{r, i} \lambda^{2} N_{W}}{2 D_{S} C_{t, i}^{0}}$, and $C_{t, i}^{H}=C_{t, i}\left(x, t=t_{H}\right)$ is the $\mathrm{H}$ filled trap profile after exposure to $\mathrm{H}$ plasma for time $t_{H}$. Figure 2 shows $C_{t, i}^{D}$ after isotope exchange (green curve) with $t_{D}=5000 \mathrm{~s}$ and $t_{H}=3000 \mathrm{~s}$. To obtain the total D profile after isotope exchange, the sum of all $i$ solutions to (9) represent the remaining D inventory.

\section{Results and discussion}

The observed D retention profile (fig. 3) is similar to other retention experiments with this W material and plasma exposure [2]. To replicate the NRA data with the model, the solutions of equation (3) with ( $t=t_{D}$, the D exposure time) for each trap site energy (defined by 1 of 3 fitting parameters for each defect type, $C_{t, i}^{0}$, and literature values of $E_{t, i}$ for the $i^{\text {th }}$ trapping energy) were added together to obtain the total D retention profile. Comparing the individual filled trap concentration profiles, the model indicates that GB traps fill much slower than the higher energy traps in dislocations and bulk vacancies even though there are more GB traps throughout the material. This is because low trapping energies have higher release rates, so these traps take more time to fill. Higher energy traps fill throughout the 
material much faster because it is rarer for an atom to escape due to thermal vibrations in the lattice.

Although the distribution of trap concentrations were free parameters used for fitting, we observe in Fig. 3 that the model represents the D depth profile data well. If these defect concentrations could be estimated accurately or measured elsewhere, this model could provide a fully predictive model for retention. However, exact knowledge of the material's defect history is not available at this point in time. Having fit the model to the D depth profile obtained for the D plasma exposed sample, the parameters $C_{t, i}^{0}$ are now fixed and can be used to model the effects of $\mathrm{H}$ isotope exchange.

We use equations (3) and (9) to estimate the loss of D after exposure to $\mathrm{H}$ plasma. Figure 4 shows the $\mathrm{D}$ depth profile after various fluences of $\mathrm{H}$ plasma as well as the resulting model predictions. The expected experimental result [2-4] of D inventory decreasing with increasing $\mathrm{H}$ fluence is again observed here. The model follows the isotope exchange data fairly well with the two lowest $\mathrm{H}$ fluences, except for the data point closest to the surface which could be accounted for by blistering or other surface morphology effects not considered in the model. For the highest $\mathrm{H}$ fluences, it seems that the model predicts a faster rate of exchange and exchanges deeper within the material. The overestimation could be due to the assumption that an exchanged D disappears immediately from the material, where realistically it could be re-trapped before reaching the surface.

Atoms can escape more easily (i.e. exchange atoms more efficiently) from lower energy traps, and we observe this in the model. The D concentration profiles resulting from lower energy traps are reduced more rapidly during isotope exchange than the concentration profiles from higher energy traps. These rapidly exchanging D profiles produce an odd result for the total predicted $\mathrm{D}$ concentration profile near the surface by appearing to create oscillating features. These features arise because each trapping site is treated separately in 
the model. Physically, these trapped concentration profiles are not independent of each other: D atoms released from a low energy trap could then be re-trapped by a higher energy trap. Overall this model gives qualitative agreement with what is experimentally observed; however, re-trapping of solute $\mathrm{D}$ atoms during isotope exchange must still be added.

\section{Summary}

A retention model is proposed that models how trapping sites, defined by their trapping energy and their distribution in the material, fill up as $\mathrm{H}$ isotopes diffuse through solution. Three defect types are used in the development of the model: grain boundary vacancies, dislocations, and bulk vacancies. The trapping energies are defined by DFT modeling [12-14]. We assume these 3 defect types are uniformly distributed in the material, and since exact knowledge of their distributions are not known, we consider their values as 3 free parameters. Each trapping site is treated separately allowing insight into how each site accumulates and exchanges inventory with isotopes. Another advantage of the model is that it is now feasible to include any number of trapping types, which will allow us to include defects not intrinsic in the material like ion or neutron damage induced vacancies. We reproduce the experimental $\mathrm{D}$ retention data well and capture the main physics of isotope exchange with the model. This recasting of the Doyle isotope exchange model [5] with a $\mathrm{H}$ diffusion arrival rate seems to agree well with the data at low $\mathrm{H}$ fluences, but seems to overestimate the isotope exchange rate at high $\mathrm{H}$ fluences. Future work will not include the over-simplification of $\mathrm{D}$ atoms disappearing from the material after exchange. We observe that the Doyle model must include re-trapping and diffusion in materials like $\mathrm{W}$ to better estimate the isotope exchange rate.

\section{Acknowledgements}

This work was supported by the US Department of Energy under DE-SC0001999 and DE-FG02-07ER54912 and the University of California Office of President Research Fund 
under Award Number 12-LR-237801. The ion beam analysis work at IBML was supported by a LANL new program development grant in support the UCOP project.

\section{References}

[1] J.Roth et al, Plasma Phys. Control. Fusion 50 (2008) 103001 (20pp)

[2] J.L.Barton et al, Nucl. Instr. and Meth. B 332 (2014) 275-279

[3] J.Roth et al, J. Nucl. Mater. 432 (2013) 341-347

[4] V.Kh. Alimov et al, Phs. Scr. T145 (2011) 014037 (5pp)

[5] B.L.Doyle et al, J. Nucl. Mater. 93 (1980) 551-557

[6] PLANSEE Metall GmbH - High Performance Materials, A-6600 Reutte, Austria. http://www.plansee.com

[7] G.R. Tynan, et al, J. Vac. Sci. Technol. A 15 (1997) 2885

[8] M. Mayer et al, Nucl. Instr. and Meth. B 267 (2009) 506-512

[9] R.A. Causey, J. Nucl. Mater. 300 (2002) 91

[10] G.R. Longhurst TMAP7: ‘Tritium Migration Analysis Program', User Manual, Idaho National Laboratory, INEEL/EXT-04-02352, Rev. 2 (2008)

[11] R. Frauenfelder, J. Vac. Sci. Technol. 6 (1969) 388

[12] Y.L.Liu et al, Physical Review B 79, 172103 (2009)

[13] W.Xiao, W.T. Geng, J. Nucl. Mater. 430 (2012) 132-136

[14] H.B.Zhou et al, Nucl. Fusion 50 (2010) 025016 (10pp)

[15] M. Shimada et al, Phys. Scr. T145 (2011) 014051

\section{Figure captions}

Fig. 1. Diffusion rate much lower than trapping and release rates

Fig. 2. The solution for one trapping type before and after isotope exchange

Fig. 3. $\mathrm{D}$ retention NRA data in $\mathrm{W}$ after a $\mathrm{D}$ fluence of $10^{24}$ ions $/ \mathrm{m}^{2}$ and modeled by the sum of all of the partially filled trap concentrations for each trapping type: grain boundaries ( 5 
types uniformly distributed 0.04 at.\%), dislocations ( 6 types uniformly distributed $1.3 \times 10^{-3}$ at.\%), and bulk vacancies (10 types uniformly distributed $5 \times 10^{-4}$ at.\%).

Fig. 4. Deuterium retention profiles after isotope exchange and the corresponding isotope exchange model prediction, where $t_{H}$ is calculated from the flux and fluence of the H plasma. The D profile for the non-isotope exchanged sample is also plotted for reference 
Fig. 1.

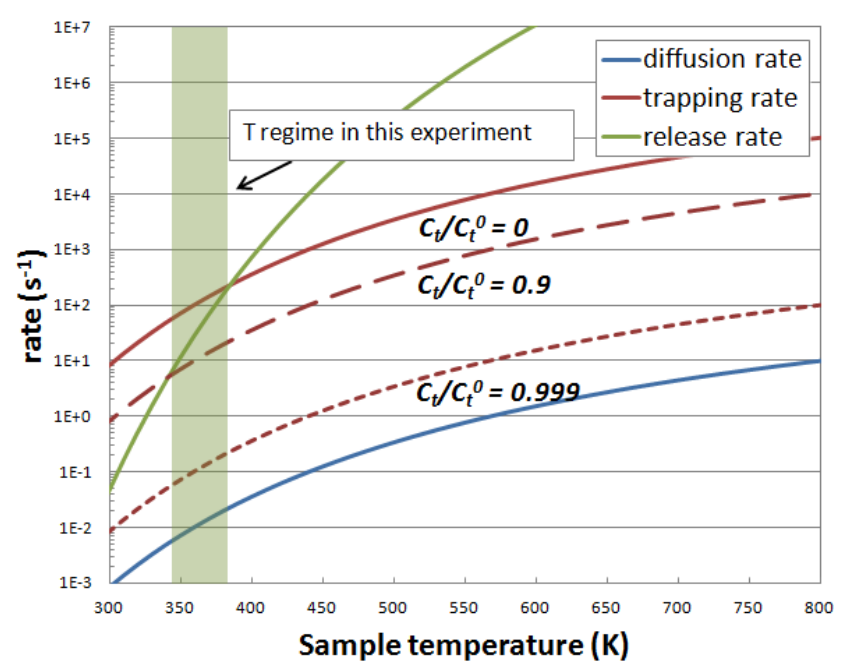


Fig. 2.

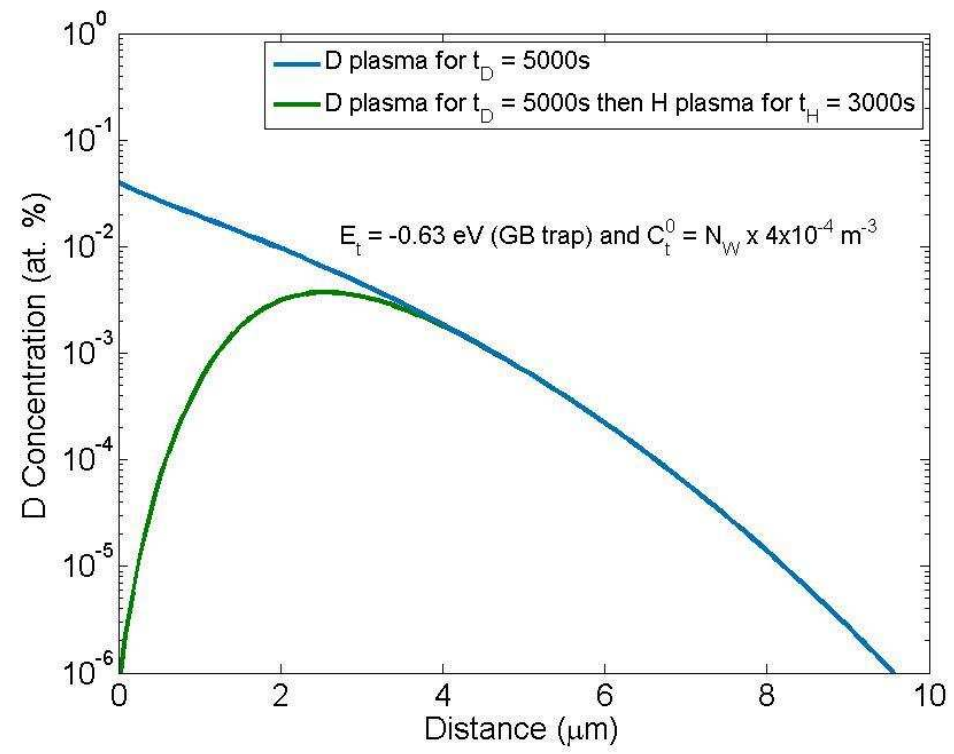


Fig. 3.

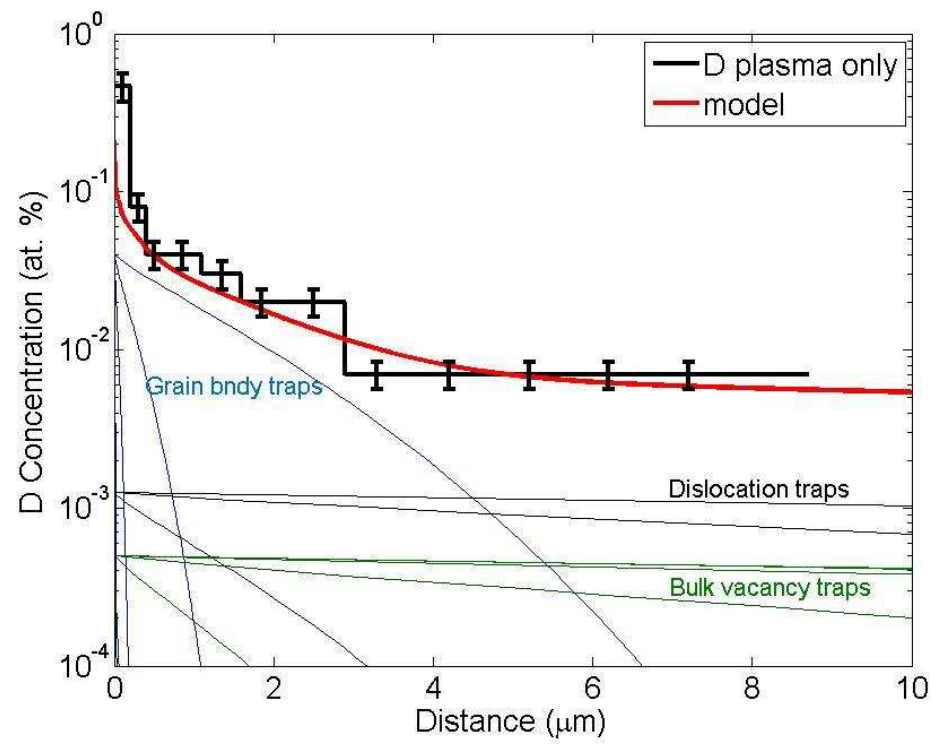


Fig. 4.
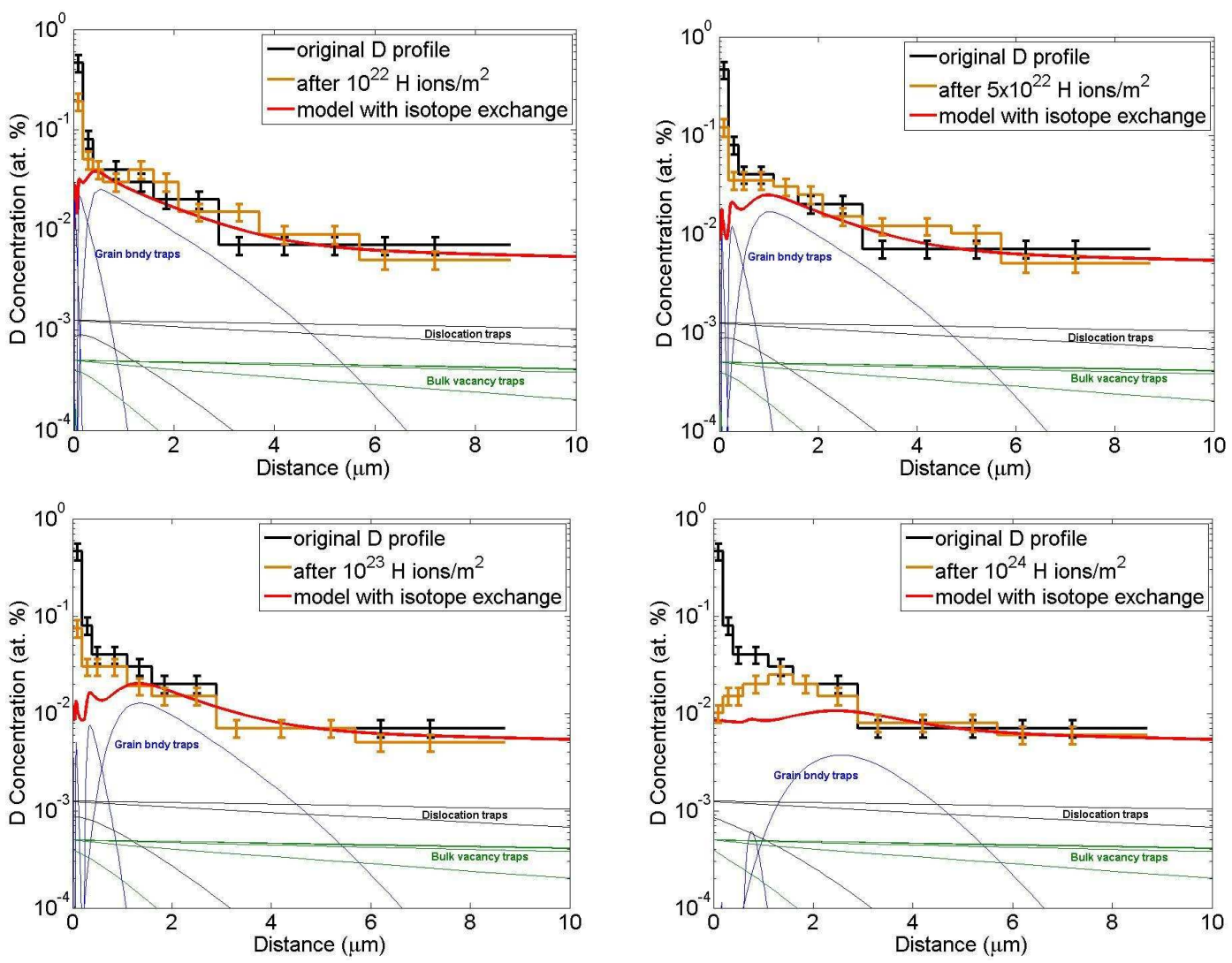